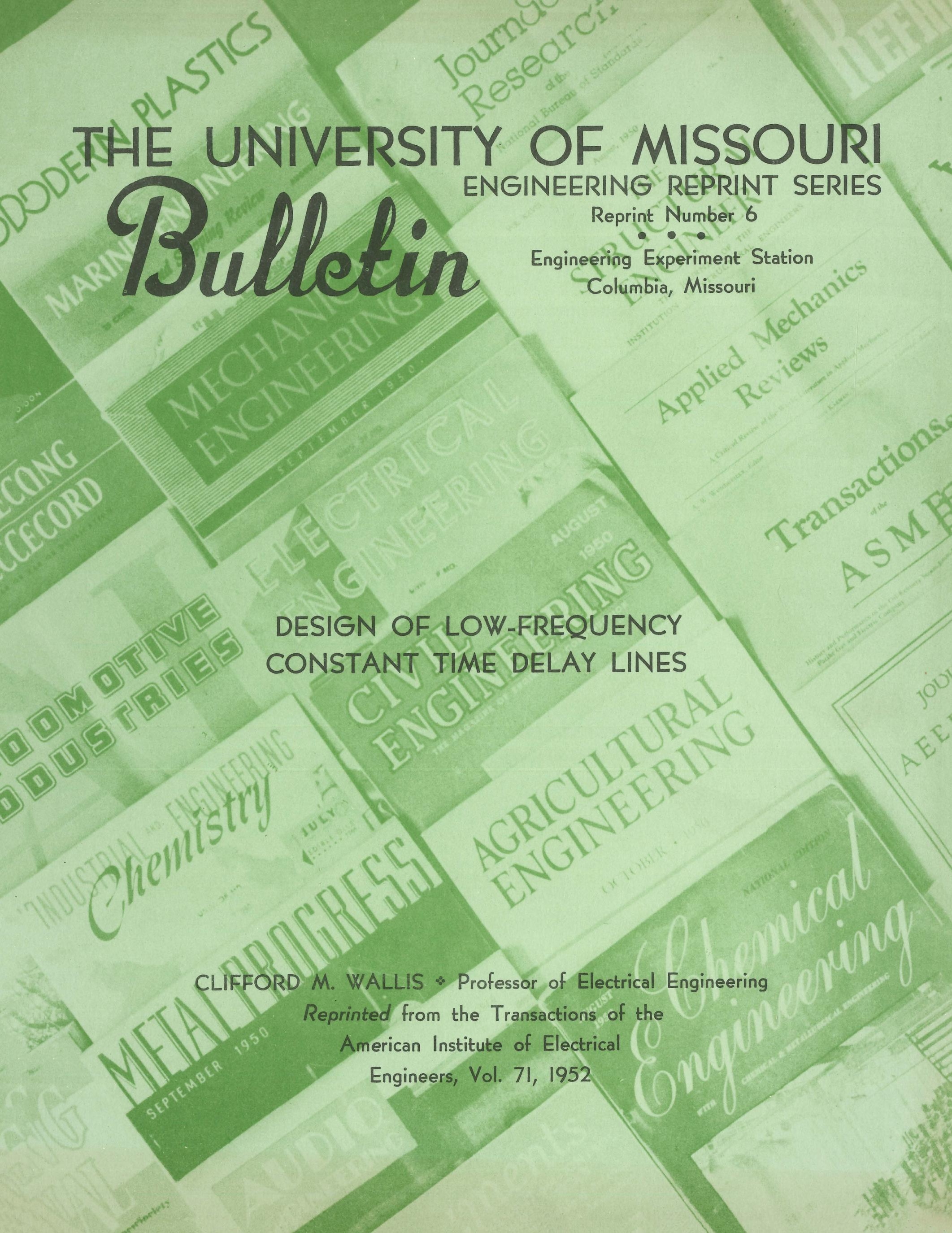




\section{COLLEGE OF ENGINEERING \\ THE ENGINEERING EXPERIMENT STATION}

The Engineering Experiment Station was organized in 1909 as a part of the College of Engineering. The staff of the Station includes all members of the Faculty of the College of Engineering, together with Research Assistants supported by the Station Funds.

The Station is primarily an engineering research institution engaged in the investigation of fundamental engineering problems of general interest, in the improvement of engineering design, and in the development of new industrial processes.

The Station desires particularly to co-operate with industries of Missouri in the solution of such problems. For this purpose, there is available not only the special equipment belonging to the Station but all of the equipment and facilities of the College of Engineering not in immediate use for class instruction.

Inquiries regarding these matters should be addressed to

\section{THE UNIVERSITY OF MISSOURI BULLETIN}

VOLUME 54, NUMBER 9 ENGINEERING EXPERIMENT STATION REPRINT SERIES, NUMBER 6

Published by the University of Missouri at Room 102, Building T-3, Columbia, Missouri. Entered as second-class matter, January 2, 1914, at post office at Columbia, Missouri, under Act of Congress of August 24, 1912. Issued four times monthly Octoier through May, three times monthly June through September. ..-175

March 1, 1953 
A Preprint from AIEE TRANSACTIONS, Volume 71, 1952. All rights reserved. Publications

Office, 500 5th Avenue, New York 36, N. Y.

Printed in the United States of America.
Published by the American Institute of Electrical Engineers. H. H. Henline, Secretary; K. B. McEachron, Chairman, Publication Committee; Charles S. Rich, Editor

\title{
Design of Low-Frequency Constant Time Delay Lines
}

\author{
CLIFFORD M. WALLIS \\ FELLOW AIEE
}

D ELAY lines are used in many applications in both high-frequency and low-frequency fields. In low-frequency applications such lines consist of a cascade connection of inductance-capacitance network sections employing lumped constants. Ideally, the line should have no attenuation; its characteristic impedance should be constant, and the phase shift should be a linear function of frequency. With the latter condition, the time delay is constant and the character of the signal is unchanged in its transmission over the line. In design practice, one attempts to meet as closely as possible these ideal conditions within the limits of cost, space, and practicability.

When the desired delay time is large, that is, in the magnitude of milliseconds and the passband ranges from zero frequency to several kilocycles, the line necessarily must be long in terms of the number of sections used. In addition to the cost, space requirements, line loss, and simplicity of construction become important factors of consideration. Generally speaking, the closer the tolerance on constancy of delay time the smaller must be the delay time per section and hence the greater the number of sections. This means larger line loss, more space, and higher cost for a fixed over-all delay time. Again, to obtain constancy of delay time over a large portion of the passband by use of a complicated network section in-

Paper 52-150, recommended by the AIEE Basic Sciences Committee and approved by the AIEE Technical Program Committee for presentation at the AIEE South West District Meeting, St. Louis, Mo., April 15-17, 1952. Manuscript submitted January 11,1952 ; made available for printing March 3, 1952.

Clifford M. Wallis is at the University of Missouri, Columbia, Mo. creases the space required and line cost. Consequently, one must determine the kind of network that best meets the electrical, space, and economic requirements of the application. It is the purpose of this paper to present data and to compare the relative merits of several types of networks that are particularly useful where essentially constant delay for signals ranging in frequency from near zero to some arbitrary upper limit is required.

Where the frequency range lies in this part of the spectrum an inductancecapacitance network of the low-pass type is employed. There are a number of these whose characteristics make them useful in delay line design. In particular, they are:

The M-derived section.

The bridged-T section.

The capacitance shunted section.

The unsymmetrical Pierce section.

Analyses of the first three have been presented in a recent article by Hebb, Horton and Jones. ${ }^{1}$ Although these circuits often are used in filter design ${ }^{2}$ their time-delay characteristics are not as commonly known. The unsymmetrical Pierce section is perhaps even less familiar. As far as the author knows, its virtue as a delay network was first discovered by G. W. Pierce around 1919, though little mention of it has been made in the literature. The major portion of this discussion will be devoted to presenting an analysis and the characteristics of this circuit. For the sake of comparison, the time-delay characteristics and design equations of the Mderived and bridged-T sections will be included. The latter two symmetrical sections and the Pierce unsymmetrical section are shown in Figures 1(A), 1(B), and $1(\mathrm{C})$ respectively. All are of the $\mathrm{T}$ - type structure employing mutual coupling between the inductive elements of the T.

The following symbols are used in the analysis:

$Z_{c}=$ characteristic impedance

$Z_{c o}=$ characteristic impedance at $f \rightarrow 0$

$\omega=2 \pi f$

$\beta=$ phase-shift angle

$f_{c}=$ frequency at which $\beta=180$ degrees

$T_{g}=$ group delay time $=d \beta / d \omega$

$T_{o}=$ delay time at $f \rightarrow 0$ (called zero-frequency delay time)

$k=$ coupling coefficient

$Z_{1} Z_{2}=$ iterative impedances of unsymmetrical section in forward and backward direction

Group delay time represents the delay time for a narrow band of frequencies centered about the angular frequency $(\omega)$. Defined in this manner it is the slope of the phaseshift curve.

The conventional assumption is made that the coils and capacitors are lossless components as far as determining the delay and impedance characteristics of the section.

In designing a delay line one usually starts with the following factors specified to meet a given application:

\section{Frequency range.}

2. Total delay time or delay time between feed-in points if signals are to be injected into the line at several points.

3. Nominal (zero frequency) characteristic impedance.

4. Allowable variation (tolerance) of items 2 and 3 over the specified frequency range.

It is convenient to express the delay time in terms of its zero frequency value and the per-cent variation. Therefore, the design equations are expressed in terms of this parameter, the nominal characteristic impedance, and such other parameters as are needed.

\section{M-Derived Section}

A type of M-derived section which offers an optimum time-delay characteristic in its passband is one where the two coils are connected series aiding. Its equivalent circuit is shown in Figure 2. In terms of the zero-frequency characteristic impedance and delay time, the constants of the section are 


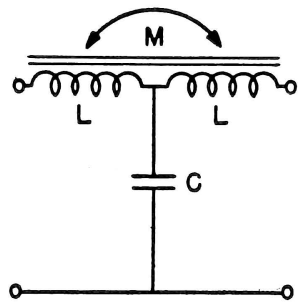

(A)

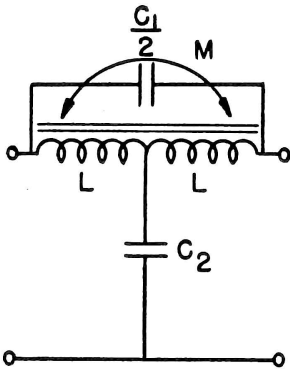

(B)

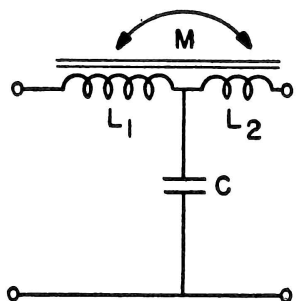

(c)
Figure 1. Three inductance-capacitance networks used in low-frequency time-delay lines

$L=\frac{T_{o} Z_{c o}}{2(1+k)}$

$C=\frac{2 L(1+k)}{Z_{c o}{ }^{2}}=\frac{T_{o}}{Z_{c o}}$

The cutoff frequency and phase-shift angle are

$f_{c}=\frac{m}{\pi T_{o}}$

$\beta=2 \sin ^{-1}\left[\frac{m u}{\sqrt{1-\left(1-m^{2}\right) u^{2}}}\right]$

where $m=\sqrt{(1+k) /(1-k)}$ and $u=f / f c$. The group delay time at any frequency within the passband is

$T_{g}=\frac{m}{\pi f_{c} \sqrt{1-u^{2}}\left[1-\left(1-m^{2}\right) u^{2}\right]}$

Normalizing with respect to $T_{o}$.

$\frac{T_{g}}{T_{o}}=\frac{1}{\sqrt{1-u^{2}}\left[1-\left(1-m^{2}\right) u^{2}\right]}$

Curves of $\left(T_{g}-T_{o}\right) / T_{o}$ are plotted in Figure 3 for several values of coupling coefficient. Included is the one for $k=0$, representing the constant- $\mathrm{K}$ type of sec- tion. For values of $k$ in the neighborhood of 0.25 we see that the delay time remains essentially constant over approximately 60 per cent of the passband. The serious disadvantage of this type of section lies in the difficulty of obtaining a coupling coefficient this low when the two coils are wound on a common toroidal core of magnetic material. To obtain a value of $k=0.25$, and at the same time maintain high coil $Q$ 's, would demand a special arrangement of coils.

\section{Bridged-T Section}

Though requiring an extra component, the bridged- $T$ circuit has better delay characteristics for high values of $k$ than the M-derived section. The equivalent electric circuit is shown in Figure 4 for the coils connected series aiding. The phase shift in the passband is

$$
\begin{aligned}
\beta=2 \tan ^{-1}\left[u_{2} \sqrt{\frac{1+k}{1-k}} \times\right. & \left.\frac{1}{\sqrt{1-\left(u_{1}^{2}+u_{2}^{2}\right)+u_{1}^{2} u_{2}^{2}}}\right]
\end{aligned}
$$

where $u_{1}=f / f_{1}$

$u_{2}=f / f_{2}$

and

$f_{1}=\frac{1}{2 \pi \sqrt{C_{1} L(1+k)}}$

$f_{2}=\frac{\sqrt{2}}{2 \pi \sqrt{C_{2} L(1-k)}}$

For the characteristic impedance we write

$Z_{c}=\sqrt{\frac{2 L(1+k)}{C_{2}}} \sqrt{\frac{1-\left(u_{1}^{2}+u_{2}^{2}\right)+u_{1}^{2} u_{2}^{2}}{\left(1-u_{1}^{2}\right)^{2}}}$

From equation 7 one may obtain the expression for the delay time ratio as

$$
\begin{aligned}
& \frac{T_{g}}{T_{o}}=\frac{1-u_{1}{ }^{2} u_{2}{ }^{2}}{\left[1-u_{1}{ }^{2}+\left(\frac{2 k}{1-k}\right) u_{2}{ }^{2}+u_{1}{ }^{2} u_{2}{ }^{2}\right] \times} \\
& \sqrt{1-\left(u_{1}{ }^{2}+u_{2}{ }^{2}\right)+u_{1}{ }^{2} u_{2}{ }^{2}}
\end{aligned}
$$

In general there are two frequencies at which the phase-shift angle $\beta$ is equal to 180 degrees, indicating the presence of two passbands. These frequencies are $f_{1}$ and $f_{2}$. If we let $a=f_{1}{ }^{2} / f_{2}{ }^{2}=u_{2}{ }^{2} / u_{1}{ }^{2}$, equations 9 and 10 become

$$
Z_{c}=\sqrt{\frac{2 L(1+k)}{C_{2}}} \sqrt{\frac{1-a u_{1}^{2}}{1-u_{1}^{2}}}
$$

and

$$
\frac{T_{g}}{T_{o}}=\frac{1-a u_{1}{ }^{4}}{\left[1+u_{1}{ }^{2}\left(\frac{2 k a}{1-k}-1\right)+a u_{1}{ }^{4}\right] \times} \sqrt{\sqrt{\left.1-a u_{1}{ }^{2}\right)\left(1-u_{1}{ }^{2}\right)}}
$$

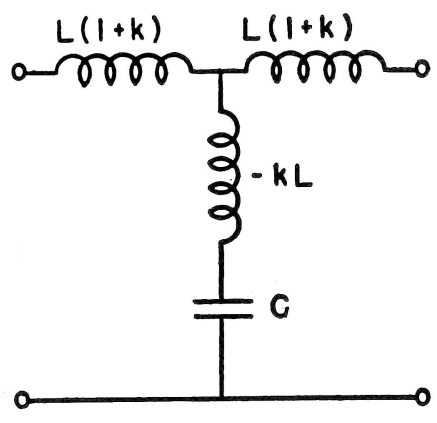

Figure 2 (above). Equivalent circuit of the $M$-derived section shown in Figure $1(A)$

Figure 3 (right). Per cent variation of delay time versus frequency ratio for $M$ derived section of Figure 1(A)

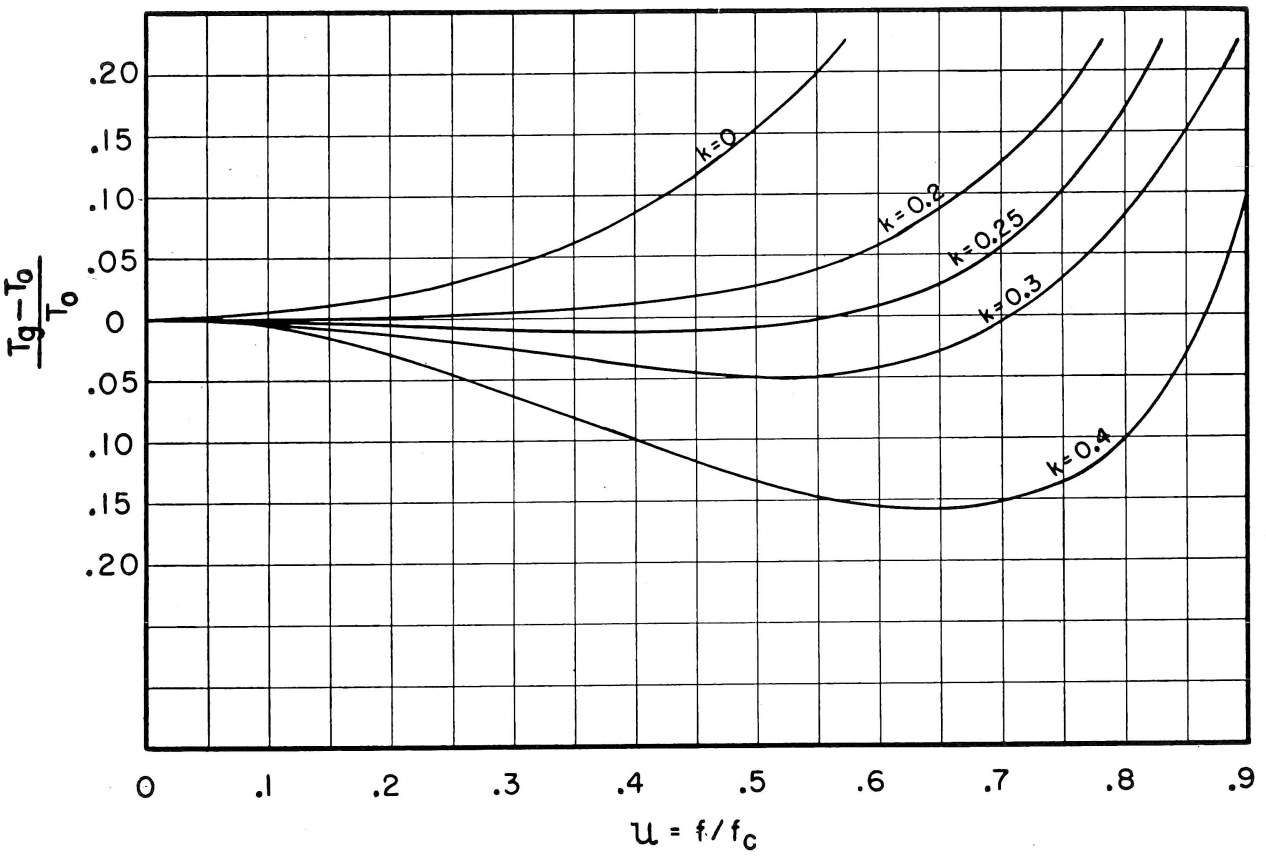




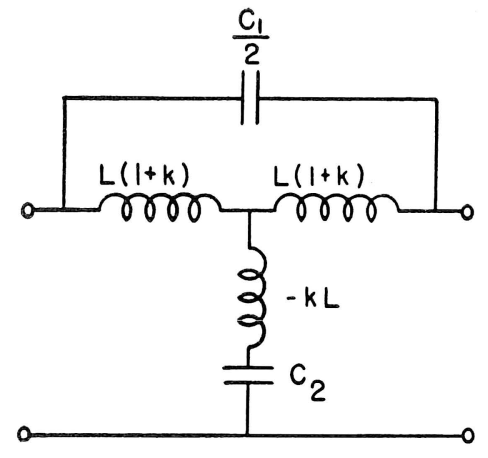

Figure 4 (above). Equivalent circuit of bridged-T section shown in Figure 1(B)

Figure 5 (right). Per cent variation of delay time versus frequency ratio for bridged-T section of Figure 1(B)

A type of inductor widely used in lowfrequency ( 1 to 50 kilocycles) delay lines is one which consists of two coils wound on a toroid core. The core is a bonded lowloss magnetic material such as molybdenum permalloy. The coil $Q$ is relatively high and, as there is little stray field, the coil units can be arranged in a compact manner. Their coupling coefficient $k$ ranges from about 0.8 to 0.9 and for the smaller sized units centers pretty well around 0.87 . With a value of $k$ in this range the parameter $a$ is less than unity and $f_{1}$ is the lower cutoff frequency. The curves of Figure 5 show that for a minimum variation of delay time the values of $a$ range between 0.11 to 0.15 , depending on the exact value of $k$.

When the allowable variation in the delay time of a line for a given frequency range is specified, along with the line impedance, reference to a family of curves such as those of Figure 5 allows one to determine the proper value of $a$. With this parameter determined, $f_{1}$ and $f_{2}$ are fixed and the constants of the section as well as the delay time per section can be calculated from the equations

$T_{o}=\frac{1}{\pi f_{2}} \sqrt{\frac{1+k}{1-k}}$

$C_{2}=\frac{T_{o}}{Z_{c o}}$

$L=\frac{T_{0} Z_{c o}}{2(1+k)}$

The capacitance $C_{1}$ is determined from equation $8(A)$.

\section{Pierce Unsymmetrical Section}

The Pierce unsymmetrical section is shown in Figure 1(C). The two coils, whose self-inductances are designated as $L_{1}$ and $L_{2}$, are connected series aiding, and are wound to have a coefficient of coupling

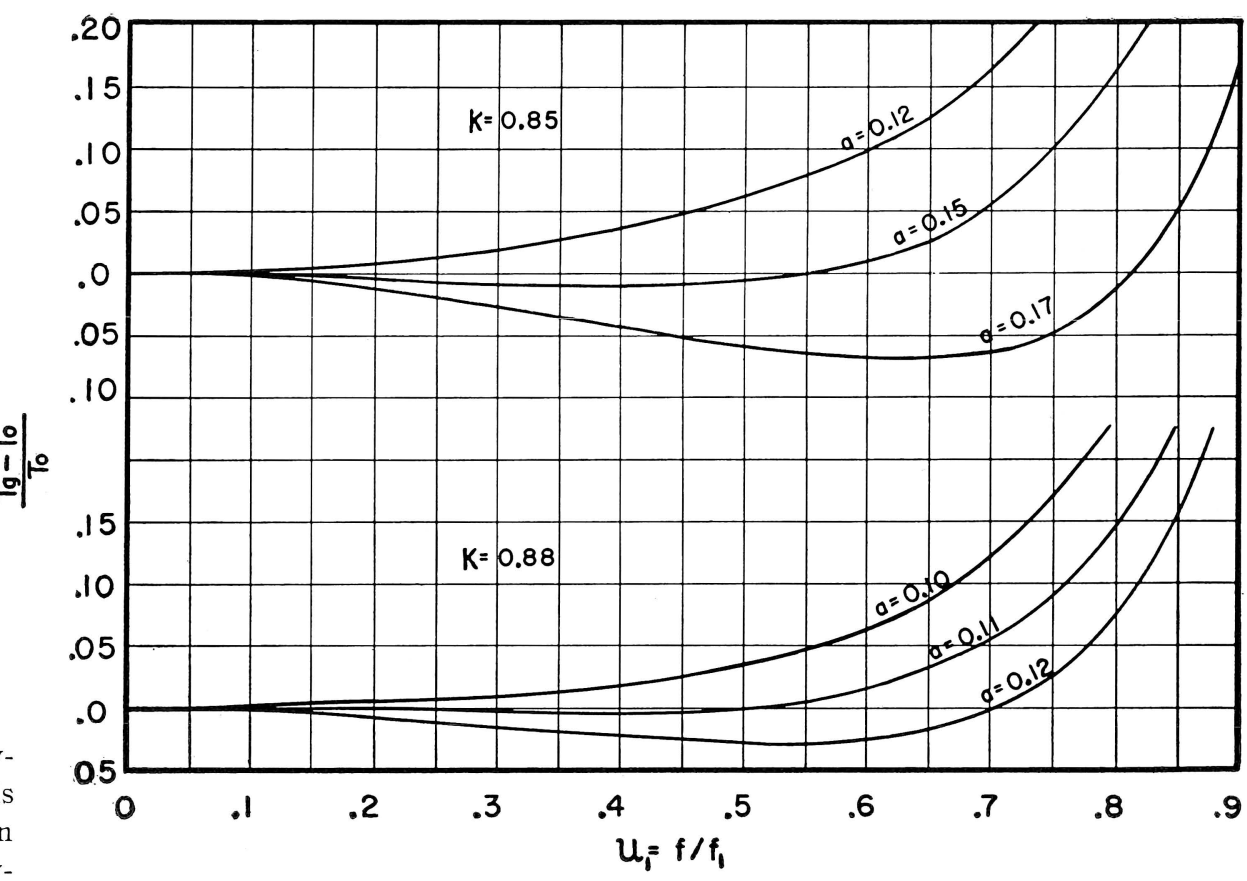

as close to unity as possible. If the ratio of $L_{1}$ to $L_{2}$ is made large and the sections are connected on an iterative basis, it is possible to secure a delay time that is nearly constant over a large portion of the passband. The avoidance of the need for loose coupling between the two coils is a distinct advantage of the Pierce section over the symmetrical M-derived type.

When connected in an iterative manner all the sections are oriented in the same direction. If each end of the line is terminated in an impedance that takes the place of an infinite continuation of the line, it is said to be terminated on an iterative basis. These impedances, designated as $Z_{1}$ and $Z_{2}$, are called the iterative impedances of the section. ${ }^{3}$

The equivalent circuit is shown in Figure 6. In terms of the circuit constants the iterative impedances are

$Z_{1} \mid=\frac{1}{2} X$

$\left\{\sqrt{\left(L_{1}+L_{2}+2 M\right)\left[4 S-\omega^{2}\left(L_{1}+L_{2}-2 M\right)\right]} \pm\right.$ $\left.j\left(L_{1}-L_{2}\right) \omega\right\}$

It may be noted that within the passband $Z_{1}$ and $Z_{2}$ are complex conjugate impedances. For frequencies in this region the phase-shift angle can be expressed either as

$\beta=2 \sin ^{-1}\left[\frac{\omega}{2} \sqrt{\frac{L_{1}+L_{2}+2 M}{S+M \omega^{2}}}\right]$

(17A)

or

$\beta=2 \tan ^{-1}\left[\omega \sqrt{\frac{L_{1}+L_{2}+2 M}{4 S-\omega^{2}\left(L_{1}+L_{2}-2 M\right)}}\right]$

For the delay time we may write

$$
\begin{aligned}
T_{0} & =\left[\frac{S}{S+M \omega^{2}}\right] \times \\
& \sqrt{\frac{4\left(L_{1}+L_{2}+2 M\right)}{4\left(S+M \omega^{2}\right)-\omega^{2}\left(L_{1}+L_{2}+2 M\right)}}
\end{aligned}
$$

There is one other factor of interest, particularly for applications where signals are to be injected into the line at several points. This is the impedance looking into the section between ground side and the point of the capacitor connection beminated on the iterative basis, this impedance is largely resistive for frequencies within a major portion of the passband. Hence, this is the point where signals are most conveniently fed into the line if bridging generators are used. This impedance, denoted by $Z_{T}$ in Figure 7 , is

$$
\begin{array}{r}
Z_{T}=\left[\frac{S^{2}}{S+M \omega^{2}}\right] \sqrt{\frac{L_{1}+L_{2}+2 M}{4 S-\omega^{2}\left(L_{1}+L_{2}-2 M\right)}}- \\
j \omega \frac{M S}{S+M \omega^{2}}
\end{array}
$$

The zero-frequency values of the several impedances and of the delay time are evident from examination of the preceding equations. In particular it may be seen that $2 Z_{T o}=Z_{10}=Z_{20}=Z_{c o}$.

Again it is convenient to set up the dequency values of the delay time and iterative impedance, the coupling coefficient $k=M / \sqrt{L_{1} L_{2}}$, and the parameter $r$, which is the ratio $L_{1} / L_{2}$. Expressed in this way

$f_{c}=\frac{1}{\pi T_{o}} \sqrt{\frac{r+2 k \sqrt{r}+1}{r-2 k \sqrt{r}+1}}$ tween coils. When the section is tersign equations in terms of the zero-fre- 


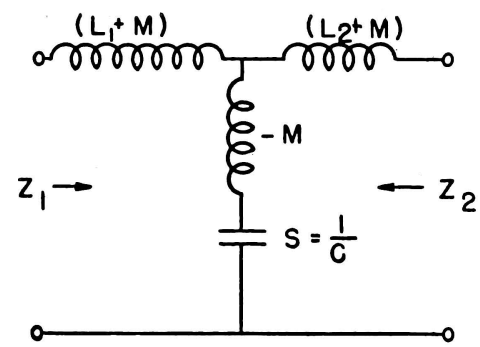

Figure 6 (above). Equivalent circuit $h^{\circ}$ of the Pierce unsymmetrical section shown in Figure 1(C)

Figure 8 (right). Per cent variation of delay time versus frequency ratio for Pierce unsymmetrical section

$L_{2}=\frac{Z_{c o} T_{o}}{r+2 k \sqrt{r}+1}$

and

$C=\frac{1}{S}=\frac{T_{o}}{Z_{c o}}$

The delay time, normalized with respect to $T_{o}$, is

$\frac{T_{g}}{T_{o}}=\frac{r-2 k \sqrt{r}+1}{\left[r-2 k \sqrt{r}+1+4 k \sqrt{r} u^{2}\right] \sqrt{1-u^{2}}}$

where $u=f / f c$. The impedance expression becomes

$\begin{gathered}Z_{1} \\ Z_{2}\end{gathered} \mid=Z_{c o}\left[\sqrt{1-u^{2}} \pm j \frac{(r-1) u}{\sqrt{r^{2}+r\left(2-4 k^{2}\right)+1}}\right]$

Minimum variation of delay is secured when $r$ is large and $k$ approaches unity. For values of $k \geq 0.95$ the optimum values of $r$ are above 50 . With this magnitude

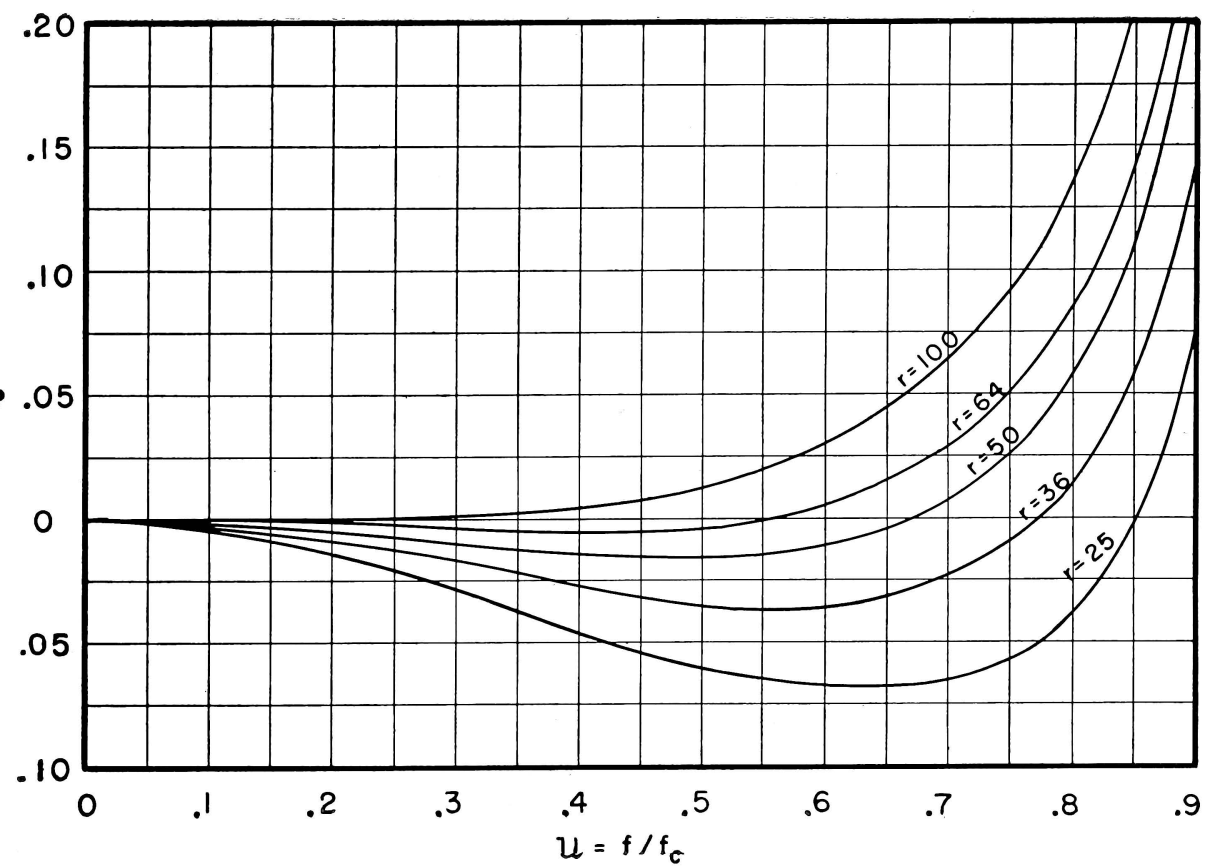

very little error is made in simplifying the preceding equations by assuming $k=1$. Whence

$f_{c}=\frac{1}{\pi T_{o}}\left[\frac{\sqrt{r}+1}{\sqrt{r}-1}\right]$

$L_{2}=\frac{Z_{c o} T_{o}}{(\sqrt{r}+1)^{2}}$

for

$\frac{T_{g}}{T_{o}}$

$$
\left.\frac{(\sqrt{r}-1)^{2}}{=\left[4 \sqrt{ } r u^{2}+(\sqrt{r}-1)^{2}\right]} \sqrt{\sqrt{1-u^{2}}}\right)
$$$$
k \geq 0.9
$$$$
r \geq 50
$$

Expressions for the iterative and bridging impedances, normalized with respect to $Z_{c o}$ become

$$
\begin{gathered}
Z_{1} / Z_{c o} \\
Z_{2} / Z_{c o}
\end{gathered} \mid=\sqrt{1-u^{2}} \pm j u
$$

$$
\frac{2 Z_{T}}{Z_{c o}}
$$

$$
\left.\begin{array}{c}
=\frac{1}{\left[1+\frac{4 \sqrt{r}}{(\sqrt{r}-1)^{2}} u^{2}\right] \times} \sqrt{\sqrt{1-u^{2}}} \\
-j \frac{u}{\frac{r-1}{4 \sqrt{r}}+\left(\frac{\sqrt{r}+1}{\sqrt{r}-1}\right) u^{2}}
\end{array}\right\} \begin{aligned}
& \text { For } \\
& k \geq 0.95 \\
& r \geq 50.0
\end{aligned}
$$

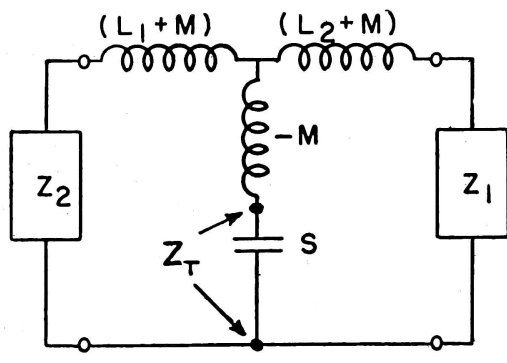

Figure 7 (above). Equivalent circuit to determine the shunt input impedance $Z_{T}$

Figure 9 (right). Cutoff frequency versus zero-frequency delay time

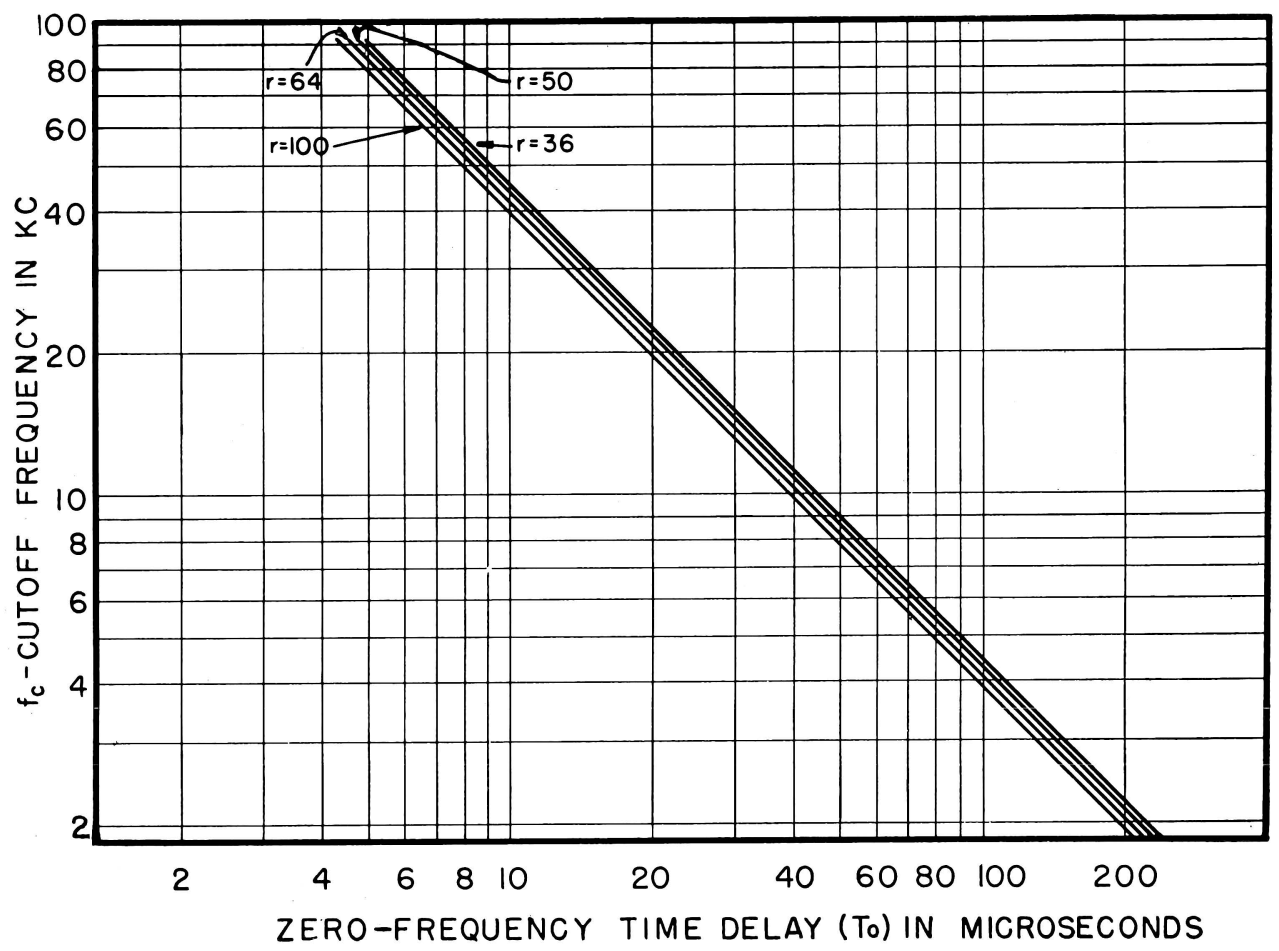



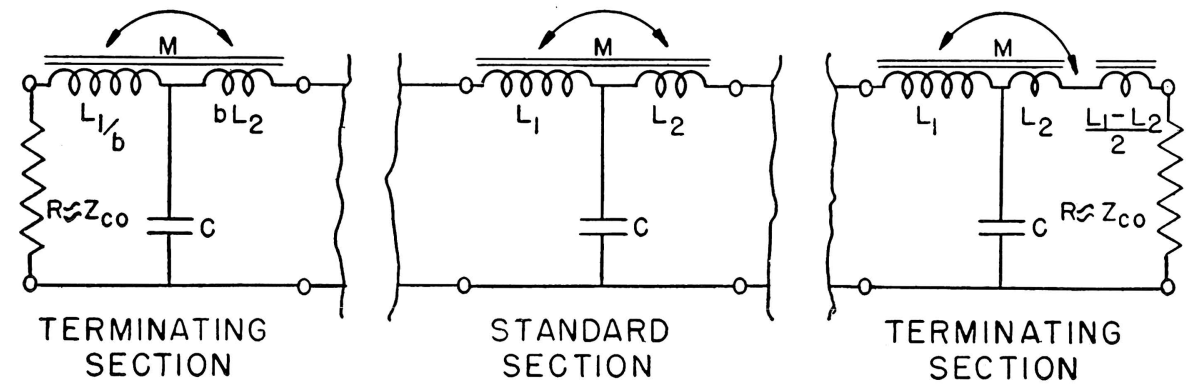

Figure 10. A method of terminating the Pierce delay line

Values of $\left(T_{g}-T_{o}\right) / T_{o}$ are plotted as functions of $u$ in Figure 8 for several values of the parameter $r$. It may be seen that with a value of $r=64$, for example, the delay time is constant to within \pm 1 per cent over 60 per cent of the passband.

Equation 28 shows that the scalar magnitude of either iterative impedance is equal to $Z_{c o}$ throughout the passband; only the resistive and reactive components vary. Figure 9 shows plots of cutoff frequency $f_{c}$ versus the delay time $T_{o}$ for several values of $r$. Using log-log scales gives straight line plots that are useful for quick reference.

\section{Termination}

Terminating delay lines that use symmetrical sections presents no serious difficulty. A resistance is chosen which best matches the characteristic impedance over the operating frequency range. However, the fact that the Pierce unsymmetrical section has iterative impedances that are complex conjugates somewhat complicates the problem of securing proper termination of a line using this type of section.

At the right end of the line, referring to Figure 10 , the terminal impedance should be the iterative impedance $Z_{1}$ which is inductive. Proper termination in this case is simply obtained by the use of an extra coil whose inductance is $\left(L_{1}-\right.$ $\left.L_{2}\right) / 2$ and a resistance that gives the best over-all match to the resistive component of $Z_{1}$. In Figure 10 the extra coil is shown as part of the terminating section on the right.

On the left end of the line, the terminal impedance should be $Z_{2}$ which has a negative reactive component $-j \omega\left(L_{1}-\right.$ $\left.L_{2}\right) / 2$. Such a reactance cannot be produced by a simple circuit. Over a limited frequency range it can be simulated fairly well by use of a parallel resistancecapacitance network with proper selection of components. Better termination is secured by the method indicated in Figure 10. The last section is terminated in $Z_{c o}$

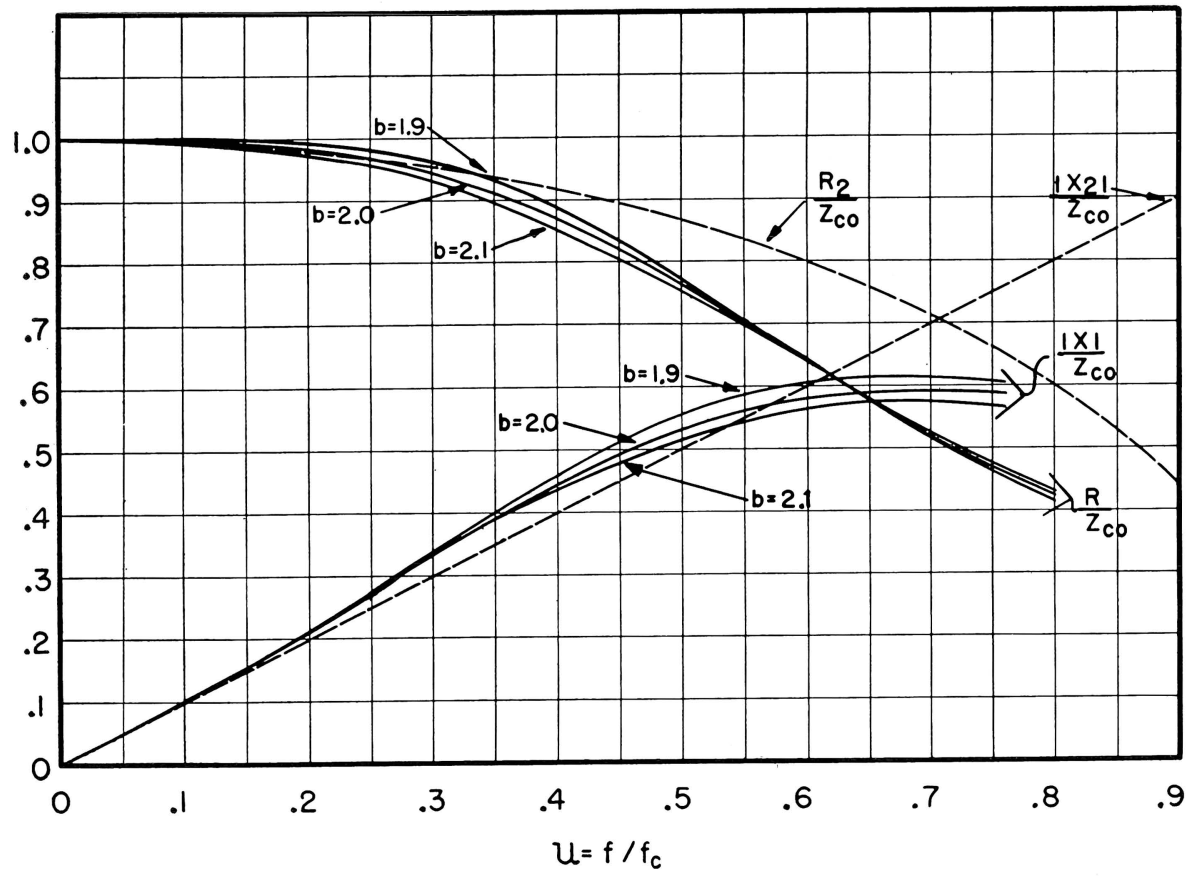

Figure 11. Curves showing degree of matching obtained by use of terminating section on left end of Pierce line as shown in Figure 10 and its coil inductances $L_{1}$ and $L_{2}$ are respectively decreased and increased by an equal ratio (designated as b) from their values in the standard line section. The coupling is made as near unity as possible as in the standard line section. Then for values of $b$ close to 2.0 the impedance looking into this section approximates $Z_{2}$ over a considerable portion of the passband. The equations for the resistive and reactive components of this impedance normalized with respect to $Z_{c o}$ are

$\frac{R}{Z_{c o}}=\frac{\left[\frac{(\sqrt{r}-1)^{2}}{4 \sqrt{r}}+u^{2}\right]^{2}}{\frac{(r-1)^{2}}{4 r} u^{2}+\frac{r}{4}\left[\frac{2}{b} u^{2}-\frac{(\sqrt{r}-1)^{2}}{2 r}\right]^{2}}$

$\frac{X}{Z_{c o}}=-j u\left[\frac{\sqrt{r}-1}{\sqrt{r}+1}\right] \times$

$\left[\frac{(\sqrt{r}-1)^{2}}{8 r}\left(r+1-b-\frac{r}{b}\right)\right]+$

$\left[\frac{r}{2 b}+\frac{1}{2}+\frac{\sqrt{r}}{b}-\frac{b}{2 r}(\sqrt{r}+1)^{2}\right] u^{2}$

$\frac{(r-1)^{2}}{4 r} u^{2}+\frac{r}{4}\left[\frac{2}{b} u^{2}-\frac{(\sqrt{ } r-1)^{2}}{2 r}\right]^{2}$

where $r$ is the ratio $-L_{1} / L_{2}$ used in the standard line section.

The curves of Figure 11 show the degree of approximation to $Z_{2}$ that can be realized with such a terminating section when $r=64$. A value of $b=2.0$ appears to give optimum matching considering both resistive and reactive components. Lines built with this termination have shown remarkably good characteristics, that is, constancy of delay time and smooth line operation.

\section{Conclusion}

In an application where the required delay time is large, necessitating a line of many sections, and space is at a premium, the Pierce section offers distinct advantages. Only two components per section are needed-one capacitor and a doublewinding inductor. The section constants are of sizes that are procured easily in practice. For example, for a 1,000-ohm 50 -microsecond section, $C=0.05$ microfarad, $L_{2}=0.61$ millihenry. With $r=64$, $L_{1}=39.5$ millihenrys.

Its time-delay characteristic compares very favorably with the other types mentioned. Although the bridged-T and capacitor-shunted types can be made to give constant delay over a slightly greater percentage of the passband, each requires an extra capacitor. For constant time delay the M-derived type demands a low coupling coefficient between coils of the inductor. This is not easily obtained where magnetic cores are used. 


\section{References}

1. On Design of Networks for Constant Time Delay, M. H. Hebb, C. W. Horton, F. B. Jones.

Journal of Applied Physics (New York, N. Y.), volume 20 , June 1949 , pages $616-20$.

2. Electric Circuits and Wave Filters (book),

A. T. Starr. Sir Isaac Pitman and Sons, London,

England, 1934

3. Communication Networks, Volume II (book), E. A. Guillemin. John Wiley and Sons, Inc., New York, N. Y., 1935, page 162.

\section{No Discussion}






\section{PUBLICATIONS OF THE ENGINEERING REPRINT SERIES}

Reprint

No.

*1. Response of Circuits to Steady-State Pulses, by D. L. Waidelich, Professor of Electrical Engineering. Reprinted from the Proceedings of the I. R. E., Vol. 37, No. 12, December 1949.

*2. Heat Transfer to Water Boiling Under Pressure, by E. A. Farber, Graduate Student, now Assistant Professor of Mechanical Engineering University of Wisconsin, and R. L. Scorah, Professor of Mechanical Engineering. Reprinted from The Transactions of The A. S. M. E., May 1948.

*3. Steady-State Waves on Transmission Lines by D. L. Waidelich, Professor of Electrical Engineering, (1950).

4. Theory of the Adiabatic Bubble, by Ralph Scorah. Reprinted from the Proceedings of the Midwestern Conference on Fluid Dynamics, J. W. Edwards, Ann Arbor, 1951.

5. Equivalent Load method for Analyzing Prestressed Concrete Structures, by Robert B. B. Moreman, Professor of Civil Engineering. Reprinted from Journal of the American Concrete Institute Vol. 23, January, 1952.

6. Design of Low Frequency Constant Time Delay Lines, by C. M. Wallis, Reprinted from Transactions American Institute of Electrical Engineers, Vol. 71, 1952. 



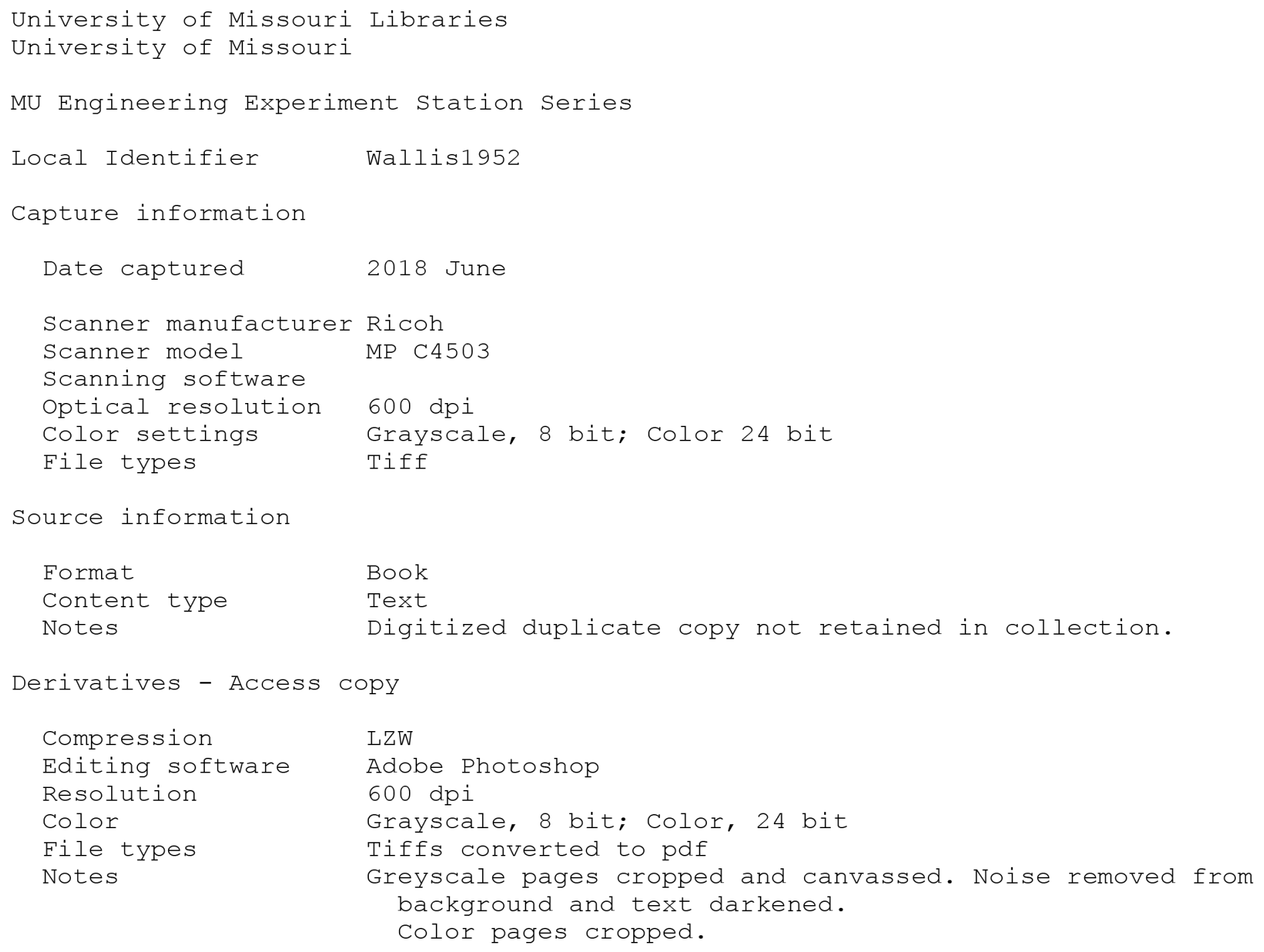

